\title{
Oxidative stress induces cell death partially by decreasing both mRNA and protein levels of nicotinamide phosphoribosyltransferase in differentiated PC12 cells
}

\author{
Cuiyan Zhou ${ }^{1}$, Weihai Ying ${ }^{\text {Corresp. } 1}$ \\ ${ }^{1}$ Med-X Research Institute and School of Biomedical Engineering, Shanghai Jiao Tong University, Shanghai, China \\ Corresponding Author: Weihai Ying \\ Email address: weihaiy@sjtu.edu.cn
}

Background. Multiple studies have indicated crucial roles of $\mathrm{NAD}^{+}$deficiency in several neurological diseases and aging. It is critical to discover the mechanisms underlying the $\mathrm{NAD}^{+}$deficiency. Decreased level of Nicotinamide phosphoribosyltransferase (Nampt) - an important enzyme in the salvage pathway of $\mathrm{NAD}^{+}$synthesis - has been found under certain pathological conditions, while the mechanisms underlying the Nampt decrease are unclear. The purpose of this study is to test the hypothesis that oxidative stress can produce decreased Nampt, and to investigate the biological effects of Nampt on $\mathrm{NAD}^{+}$synthesis and cell survival under both basal and oxidative stress conditions.

Methods. We used differentiated PC12 cells as a cellular model to investigate the effects of oxidative stress on the levels of Nampt. Multiple assays, including flow cytometry-based cell death assays and $\mathrm{NAD}^{+}$assays were conducted.

Results. First, oxidative stress can decrease the levels of Nampt mRNA and Nampt protein; second, Nampt plays significant roles in $\mathrm{NAD}^{+}$synthesis under both basal conditions and oxidative stress conditions; third, Nampt plays critical roles in cell survival under both basal conditions and oxidative stress conditions; and fourth, oxidative stress produced decreased NAD ${ }^{+}$levels and cell survival partially by decreasing Nampt. Collectively, our study has indicated that oxidative stress is a pathological factor leading to decreased Nampt, which plays important roles in oxidative stress-produced decreases in NAD ${ }^{+}$ levels and cell survival. Our findings have indicated major roles of Nampt in maintaining NAD ${ }^{+}$levels and cell survival under both basal and oxidative stress conditions. 
1 Oxidative stress induces cell death partially by decreasing both mRNA and protein levels of

2

3

$8 \quad \#$ : Corresponding author

9 Weihai Ying, Ph.D.

1954 Huashan Road

Shanghai, 200030, P.R. China

E-mail: weihaiy@sjtu.edu.cn

15

6 nicotinamide phosphoribosyltransferase

in differentiated PC12 cells

Cuiyan Zhou, Weihai Ying*

${ }^{1}$ Med-X Research Institute and School of Biomedical Engineering, Shanghai Jiao Tong

University, Shanghai 200030, P.R. China

Professor, School of Biomedical Engineering and Med-X Research Institute

Shanghai Jiao Tong University 


\section{Abstract}

Background. Multiple studies have indicated crucial roles of $\mathrm{NAD}^{+}$deficiency in several neurological diseases and aging. It is critical to discover the mechanisms underlying the $\mathrm{NAD}^{+}$ deficiency. Decreased level of Nicotinamide phosphoribosyltransferase (Nampt) - an important enzyme in the salvage pathway of $\mathrm{NAD}^{+}$synthesis - has been found under certain pathological conditions, while the mechanisms underlying the Nampt decrease are unclear. The purpose of this study is to test the hypothesis that oxidative stress can produce decreased Nampt, and to investigate the biological effects of Nampt on $\mathrm{NAD}^{+}$synthesis and cell survival under both basal and oxidative stress conditions.

Methods. We used differentiated PC12 cells as a cellular model to investigate the effects of oxidative stress on the levels of Nampt. Multiple assays, including flow cytometry-based cell death assays and $\mathrm{NAD}^{+}$assays were conducted.

Results. First, oxidative stress can decrease the levels of Nampt mRNA and Nampt protein; second, Nampt plays significant roles in $\mathrm{NAD}^{+}$synthesis under both basal conditions and oxidative stress conditions; third, Nampt plays critical roles in cell survival under both basal conditions and oxidative stress conditions; and fourth, oxidative stress produced decreased $\mathrm{NAD}^{+}$levels and cell survival partially by decreasing Nampt. Collectively, our study has indicated that oxidative stress is a pathological factor leading to decreased Nampt, which plays important roles in oxidative

41 stress-produced decreases in $\mathrm{NAD}^{+}$levels and cell survival. Our findings have indicated major roles of Nampt in maintaining $\mathrm{NAD}^{+}$levels and cell survival under both basal and oxidative stress 
43 conditions.

44

45 Keywords: Nicotinamide phosphoribosyltransferase; NAD ${ }^{+}$; Oxidative Stress; Cell Death; Aging.

46

47

48

49

50

51

52

53

54

55

56

57

58

59

60

61

Peer] reviewing PDF | (2020:11:55562:1:1:NEW 1 Apr 2021) 


\section{Introduction}

Cumulative evidence has domonstrated that $\mathrm{NAD}^{+}$plays significant roles in multiple biological processes (Ying 2008). Multiple studies have also suggested that $\mathrm{NAD}^{+}$deficiency is a pivotal pathological factor in numerous major diseases and pathological conditions (Ying 2008; Ying 2006; Zhang \& Ying 2019), including ischemic brain injury (Ying et al. 2007; Zheng et al. 2012), myocardial ischemia (Zhang et al. 2016), head trauma (Won et al. 2012), epilepsy (Liu et al. 2017), radiation-induced tissue injury (Sheng et al. 2012) and chemotherapy agent-induced tissue injury (Wang et al. 2014). $\mathrm{NAD}^{+}$deficiency also appears to play an important role in the aging process (Frederick et al. 2016; Mills et al. 2016; Son et al. 2016), which has been the biological basis for the wide applications of $\mathrm{NAD}^{+}$and $\mathrm{NAD}^{+}$precursors for delaying aging. It has critical theoretical and medical importance to expose the impact of $\mathrm{NAD}^{+}$metabolism on pathological alterations. However, there have been no sufficient studies on this critical topic.

The salvage pathway and the de novo pathway are two major pathways for $\mathrm{NAD}^{+}$synthesis (Magni et al. 2004). The nuclear enzyme Nicotinamide mononucleotide adenylyltransferase-1 (NMNAT-1) is a crucial enzyme in the two pathway of $\mathrm{NAD}^{+}$synthesis (Magni et al. 2004). Mammals use nicotinamide as the precursor for $\mathrm{NAD}^{+}$synthesis in the salvage pathway (Revollo et al. 2007): Nicotinamide phosphoribosyltransferase (Nampt) converts nicotinamide to nicotinamide mononucleotide that can be converted to NAD ${ }^{+}$by NMNATs (Revollo et al. 2007). $\mathrm{NAD}^{+}$deficiency has been observed in a variety of disease models, which may result from poly (ADP-ribose) polymerase-1 activation or reduced Nampt activity. It has been indicated that decreased Nampt activity is an important mechanism accounting for declined $\mathrm{NAD}^{+}$synthesis: 
Decreased Nampt activity has been found in the metabolic organs that are exposed to high-fat diet (Yoshino et al. 2011), Drosophila pink1 mutants (Lehmann et al. 2017), and aging (Jokinen et al. 2017; Yoshino et al. 2011). It is necessary to investigate the potential mechanisms for the decreased Nampt levels.

Our study used PC12 cells to investigate the effects of oxidative stress on both the Nampt mRNA and Nampt protein levels. We also investigated the roles of Nampt in the cell death and $\mathrm{NAD}^{+}$metabolism under both basal and oxidative stress conditions. Our experimental results have shown that oxidative stress can produce decreased levels of Nampt, which contributes to oxidative stress-produced decreases in $\mathrm{NAD}^{+}$levels and cell survival. Our current study has also indicated that Nampt plays important roles in maintaining intracellular $\mathrm{NAD}^{+}$levels and cell survival under both basal and oxidative stress conditions.

\section{Materials and methods}

\section{Materials}

Sigma Aldrich (St Louis, Missouri, USA) was the supplier of $\mathrm{H}_{2} \mathrm{O}_{2}$ (323381) and Nicotinamide (72340). FK866 (HY-50876) and P7C3 (HY-15976) were purchased from MedChemExpress (New Jersey, USA). Nampt siRNAs and control siRNAs were purchased from GenePharma (Shanghai, China). Sangon Biotech (Shanghai, China) was the supplier of Nampt primers.

\section{Cell cultures}


Institute of Biological Sciences, Chinese Academy of Sciences (Shanghai, China). PC12 cells were plated into 12- well or 24-well cell culture plates in DMEM (Hyclone, Massachusetts, USA) containing $10 \% \mathrm{FBS}, 1 \%$ penicillin and $1 \%$ streptomycin. The cells were cultured in an incubator with $5 \% \mathrm{CO}_{2}$ and the culture temperature was $37^{\circ} \mathrm{C}$.

\section{NAD $^{+}$assay}

As previously described (Alano et al. 2004), recycling assay was conducted to determine centrifugated at 12,000 g for 5 minutes, and the supernatant was transferred to new test tubes.

$3 \mathrm{~N}$ potassium hydroxide and $1 \mathrm{M}$ potassium phosphate buffer were used to neutralize the samples to $\mathrm{pH}$ 7.2. After centrifugation, the samples were mixed with reaction buffer that contained $1.7 \mathrm{mg}$ 3-[4,5-dimethylthiazol-2-yl]-2,5-diphenyl-tetrazolium bromide (MTT), $488.4 \mathrm{mg}$ nicotinamide, $10.6 \mathrm{mg}$ phenazine methosulfate, $1.3 \mathrm{mg}$ alcohol dehydrogenase, and

$2.4 \mathrm{~mL}$ ethanol in $37.6 \mathrm{~mL}$ Gly-Gly buffer $(65 \mathrm{mM}, \mathrm{pH} 7.4)$. Ten minutes later, the $\mathrm{A}_{560 \mathrm{~nm}}$ of the samples was assessed. The final concentrations of $\mathrm{NAD}^{+}$were normalized by protein levels.

\section{Annexin V/7-AAD assay}

Flow cytometry assay was conducted to determine the levels of necrosis and apoptosis of 
125 with PBS, the cells were incubated with $100 \mu \mathrm{L}$ cold $1 \mathrm{X}$ binding buffer and $5 \mu \mathrm{L}$ Annexin V,

126 which were put in ice for 15 minutes. Finally, $200 \mu \mathrm{L} 1 \mathrm{X}$ binding buffer and $5 \mu \mathrm{L}$ 7-AAD were

127 mixed into the samples. The levels of necrosis and apoptosis of PC12 cells were measured by

128 a flow cytometer (FACSAria II, BD Biosciences).

\section{Real-Time PCR assay}

Bio, Dalian, China) according to the manufacturer's protocol. cDNA was reverse-transcribed $85^{\circ} \mathrm{C}$ for 15 seconds. SYBR Premix Ex Taq produced by Takara Bio (Dalian, China) was used

136 for conducting Quantitative RT-PCR, in which the following primers were used: Nampt (sense

137 5'- TATTCTGTTCCAGCGGCAGA $-3^{\prime}$ and anti-sense 5'-

138 GACCACAGACACAGGCACTGA -3') ; GAPDH $\quad$ (sense 5'-

139 CCTGCACCACCAACTGCTTA -3' and anti-sense 5'- GGCCATCCACAGTCTTCTGA -3').

140 The following procedure was conducted for the assays: After denaturation at $95{ }^{\circ} \mathrm{C}$ for 10

141 seconds, 40 cycles of $95^{\circ} \mathrm{C}$ for 5 seconds and $60^{\circ} \mathrm{C}$ for 30 seconds were conducted. The 142 comparative threshold cycle method was used for the data analyses. The results were 143 normalized by GAPDH mRNA level. 
After treatment, PC12 cells were washed twice with PBS. The cells were lysed in RIPA

147 buffer produced by Millipore (Temecula, California, USA) which contained $1 \mathrm{mM}$ 148 phenylmethanesulfonyl fluoride and 1\% protease inhibitor cocktail produced by CWBio 149 (Beijing, China). After centrifugation at $12,000 \mathrm{rpm}$ for 10 minutes at $4{ }^{\circ} \mathrm{C}$, the protein 150 concentrations of the samples were determined by BCA Protein Assay Kit (Pierce 151 Biotechnology, Rockford, Illinois, USA). Thirty $\mu \mathrm{g}$ of the sample was electrophoresed through 152 a $10 \%$ sodium dodecyl sulfate-polyacrylamide gel and then transferred to $0.45-\mu \mathrm{m}$ 153 nitrocellulose membranes. The blots were incubated with a polyclonal Nampt antibody from Abcam (Cambridge, UK), which was diluted in TBST containing 1\% bovine serum albumin at 1:2000 ratio, at $4^{\circ} \mathrm{C}$ overnight. Subsequently, a horse radish peroxidase-conjugated secondary antibody produced by Epitomics (Zhejiang Province, China) diluted by TBST containing 1\% bovine serum albumin at 1:3000 ratio was used for incubation of the blots for $1 \mathrm{hr}$ at room temperature. The normalization of the sample was conducted by using a Tubulin antibody produced by proteintech (Wuhan, China). Quantifications of the bands were performed by GelPro Analyzer (Media Cybernetics, Silver Spring, Maryland, USA).

\section{RNA interference}

PC12 cells at the density of approximately $40 \%$ were transfected with he scrambled control siRNA sequences and Nampt siRNA with the following sequences: Sense 5'- 
167 (Invitrogen, Carlsbad, California, USA). Each well contained $2.5 \mu 1$ lipofectamine 2000, 0.06

168 nmol siRNA sequences, $100 \mu$ Opti-MEM, and $500 \mu 1$ Dulbecco's modified Eagle medium.

169 After 6 hrs, the media was replaced by Dulbecco's modified Eagle medium containing 10\%

170 FBS.

171

Statistical analyses

173

Data were shown as mean \pm SEM, which were analyzed by one-way ANOVA or two-way

ANOVA. Student - Newman - Keuls post hoc tests were used.

175

\section{Results}

177

1. Nampt inhibitor FK866 significantly decreased the intracellular $\mathrm{NAD}^{+}$level and cell survival of PC12 cells under basal conditions

We investigated the roles of Nampt in maintaining the $\mathrm{NAD}^{+}$level of PC12 cells under

basal conditions. Nampt inhibitor FK866 was used to attenuate the activity of Nampt. Our

experimental results showed that FK866 at the concentrations of 2, 5, 10, 20, and $50 \mathrm{nM}$ produced

dose-dependent decreases in the $\mathrm{NAD}^{+}$levels (Fig. 1A), indicating a critical role of Nampt in the

$\mathrm{NAD}^{+}$synthesis under basal conditions. We also conducted FACS-based Annexin V/7-ADD assay

to evaluate the role of Nampt in the cell survival under basal conditions, showing that FK866

produced dose-dependent increases in cell death, which equals to the sum of early-stage apoptosis,

late-stage apoptosis and necrosis (Figs. 1B and 1C). 


\section{2. $\mathrm{H}_{2} \mathrm{O}_{2}$ produced significant decreases in both Nampt mRNA levels and Nampt protein}

\section{levels of PC12 cells}

We further determined the impact of oxidative stress on the levels of Nampt mRNA and Nampt protein of PC12 cells: Treatment of the cells with 0.1 or $0.3 \mathrm{mM} \mathrm{H}_{2} \mathrm{O}_{2}$ led to significant increases in the Nampt mRNA level of the cells 12 hrs after the treatment (Fig. 2A). In contrast, $\mathrm{H}_{2} \mathrm{O}_{2}$ led to decreases in the Nampt mRNA level 20 hrs after the treatment (Fig. 2A). $\mathrm{H}_{2} \mathrm{O}_{2}$ did not significantly affect the protein level of Nampt 12 hrs after the $\mathrm{H}_{2} \mathrm{O}_{2}$ treatment (Figs. 2B and 2E), while $\mathrm{H}_{2} \mathrm{O}_{2}$ led to significant decreases in the Nampt protein level both 24 hrs (Figs. 2C and 2E) and 48 hrs (Figs. 2D and 2E) after the $\mathrm{H}_{2} \mathrm{O}_{2}$ treatment.

\section{Decreased Nampt led to further decreases in the $\mathrm{H}_{2} \mathrm{O}_{2}$-produced reductions of the NAD}

\section{levels and survival of PC12 cells}

To investigate the biological consequences of the $\mathrm{H}_{2} \mathrm{O}_{2}$-produced decreases in the Nampt levels of PC12 cells, we applied both Nampt siRNA and FK866 to determine whether the decreased Nampt affect the intracellular NAD ${ }^{+}$levels and cell survival of the PC12 cells exposed to $\mathrm{H}_{2} \mathrm{O}_{2}$. The results showed that Nampt siRNA treatment led to a marked decrease in the Nampt level (Figs. 3A and 3B). Nampt siRNA treatment also significantly reduced the intracellular NAD ${ }^{+}$ level of PC12 cells under basal conditions (Fig. 3C). Moreover, the Nampt siRNA treatment further reduced the intracellular $\mathrm{NAD}^{+}$level that was decreased by $\mathrm{H}_{2} \mathrm{O}_{2}$ treatment (Fig. 3C). Our results also showed that the Nampt siRNA treatment produced increased cell death under basal conditions (Figs. 3D and 3E). $\mathrm{H}_{2} \mathrm{O}_{2}$ treatment also increased the cell death, which was further 
209

210

211

212

213

214

215

216

217

218

219

220

221

222

223

224

225

226

227

228

229

enhanced by the Nampt siRNA treatment (Figs. 3D and 3E).

We found that FK866 further reduced the intracellular $\mathrm{NAD}^{+}$level that was decreased by $\mathrm{H}_{2} \mathrm{O}_{2}$ treatment (Fig. 3F). Moreover, the FK866 treatment further enhanced the levels of the $\mathrm{H}_{2} \mathrm{O}_{2}$ produced necrosis, late-stage apoptosis and early-stage apoptosis (Figs. 3G and 3H).

\section{Nampt activator $\mathrm{P} 7 \mathrm{C} 3$ suppressed the $\mathrm{H}_{2} \mathrm{O}_{2}$-produced decreases in the $\mathrm{NAD}^{+}$levels and} cell survival of PC12 cells

To further investigate the biological consequences of the $\mathrm{H}_{2} \mathrm{O}_{2}$-produced reduction of the Nampt protein levels of PC12 cells, we used Nampt activator P7C3 to determine the roles of Nampt activation in the intracellular $\mathrm{NAD}^{+}$levels and survival of $\mathrm{PC} 12$ cells exposed to $\mathrm{H}_{2} \mathrm{O}_{2}$ : $\mathrm{P} 7 \mathrm{C} 3$ attenuated the $\mathrm{H}_{2} \mathrm{O}_{2}$-produced decrease in the intracellular NAD ${ }^{+}$level of PC12 cells (Fig. 4A). P7C3 also reduced the $\mathrm{H}_{2} \mathrm{O}_{2}$-produced increase in the cell death (Figs. 4B and 4C).

We also determined the effects of nicotinamide (NAM) - a substrate of Nampt - on both intracellular NAD ${ }^{+}$levels and cell death of $\mathrm{H}_{2} \mathrm{O}_{2}$-treated PC12 cells. NAM attenuated the $\mathrm{H}_{2} \mathrm{O}_{2}$ produced decrease in the intracellular NAD ${ }^{+}$level (Supplemental Fig. 2A). NAM also attenuated the $\mathrm{H}_{2} \mathrm{O}_{2}$-produced increase in the cell death (Supplemental Figs. 2B and 2C).

\section{Discussion}

Our study has generated the following important findings: First, Nampt plays critical roles in both $\mathrm{NAD}^{+}$synthesis and survival of PC12 cells under basal conditions; second, $\mathrm{H}_{2} \mathrm{O}_{2}$ produced significant decreases in both Nampt mRNA levels and Nampt protein levels of PC12 cells; and 
230

231

third, $\mathrm{H}_{2} \mathrm{O}_{2}$ induced cell death partially by producing the decreases in both Nampt mRNA levels and Nampt protein levels, since the Nampt siRNA and Nampt inhibitor significantly exacerbated the $\mathrm{H}_{2} \mathrm{O}_{2}$-produced decreases in the intracellular $\mathrm{NAD}^{+}$level and cell survival, while the Nampt activator attenuated the $\mathrm{H}_{2} \mathrm{O}_{2}$-produced decreases in the intracellular $\mathrm{NAD}^{+}$level and cell survival. Cumulating evidence has suggested crucial roles of impaired $\mathrm{NAD}^{+}$metabolism in both aging and multiple diseases (Ying 2008; Ying et al. 2007; Zhang \& Ying 2019), indicating that it is necessary to determine the roles of $\mathrm{NAD}^{+}$deficiency under these conditions (Ying 2008; Ying et al. 2007; Zhang \& Ying 2019). In the tissues or organs under certain pathological conditions, including the metabolic organs that are exposed to HFD (Yoshino et al. 2011), Drosophila pink1 mutants (Lehmann et al. 2017), and aging (Jokinen et al. 2017; Yoshino et al. 2011), decreased levels of Nampt have been reported. However, there is significant deficiency in the mechanisms underlying the Nampt alterations under pathological conditions.

Our current study has shown that $\mathrm{H}_{2} \mathrm{O}_{2}$ was capable of decreasing both protein levels and mRNA levels of Nampt in PC12 cells. Increased oxidative stress has been found in aging and multiple pathological conditions (Rani et al. 2016; Ureshino et al. 2014; Zuo et al. 2019). Therefore, our finding has suggested a novel mechanism regarding the Nampt decrease in aging and under multiple pathological conditions. Due to the crucial roles of $\mathrm{NAD}^{+}$deficiency in aging and numerous diseases, our finding has also suggested a novel relationship between the pathological alterations and $\mathrm{NAD}^{+}$metabolism in aging and the diseases: The oxidative stress in the diseases and aging led to decreased Nampt, producing decreased $\mathrm{NAD}^{+}$synthesis thus resulting in multiple pathological changes. 

and Nampt protein levels, which is reasonable for the following reasons: Since $\mathrm{NAD}^{+}$was involved in a variety of biological processes, oxidative stress-produced $\mathrm{NAD}^{+}$decreases can lead to cell death. For defensing oxidative stress, both Nampt mRNA levels and Nampt protein levels can be induced by oxidative stress in the early phase. When the oxidative stress last for a significant duration of time, cells may lose the capacity to maintain the Nampt mRNA levels and Nampt protein levels, leading to reductions of the mRNA and protein levels of Nampt. activator significantly attenuated the $\mathrm{H}_{2} \mathrm{O}_{2}$-induced reductions of the $\mathrm{NAD}^{+}$level and cell survival.

These observations have suggested a mechanism regarding oxidative stress-produced cell death at

least for some cell types.

There are two $\mathrm{NAD}^{+}$synthesis pathways in cells, while the effects of Nampt on the general

$\mathrm{NAD}^{+}$-generating ability and survival of various cell types under basal conditions are unclear. Our current findings have indicated that Nampt plays critical roles in both $\mathrm{NAD}^{+}$synthesis and cell survival under basal conditions in differentiated PC12 cells, since the Nampt siRNA and Nampt inhibitor FK866 produced profound decreases in both NAD ${ }^{+}$levels and survival of the cells under basal conditions.

\section{Conclusion}


274 levels and cell survival under basal conditions and oxidative stress conditions. 
Acknowledgment:

282

The authors would like to acknowledge the financial support by two research grants from

283

a Major Special Program Grant of Shanghai Municipality (Grant \# 2017SHZDZX01) (to W.Y.).

284

285

286

287

288

289

290

291

292

293

294

295

296

297

298

299

300

301

PeerJ reviewing PDF | (2020:11:55562:1:1:NEW 1 Apr 2021) 
302

303

304

305

306

307

308

309

310

311

312

313

314

315

316

317

318

319

320

321

322

\section{References:}

Alano CC, Ying W, and Swanson RA. 2004. Poly(ADP-ribose) polymerase-1-mediated cell death in astrocytes requires $\mathrm{NAD}^{+}$depletion and mitochondrial permeability transition. Journal of Biological Chemistry 279:18895-18902. 10.1074/jbc.M313329200

Frederick DW, Loro E, Liu L, Davila A, Jr., Chellappa K, Silverman IM, Quinn WJ, 3rd, Gosai SJ, Tichy ED, Davis JG, Mourkioti F, Gregory BD, Dellinger RW, Redpath P, Migaud ME, Nakamaru-Ogiso E, Rabinowitz JD, Khurana TS, and Baur JA. 2016. Loss of NAD Homeostasis Leads to Progressive and Reversible Degeneration of Skeletal Muscle. Cell Metabolism 24:269-282. 10.1016/j.cmet.2016.07.005

Jokinen R, Pirnes-Karhu S, Pietilainen KH, and Pirinen E. 2017. Adipose tissue NAD ${ }^{+}$ homeostasis, sirtuins and poly(ADP-ribose) polymerases -important players in mitochondrial metabolism and metabolic health. Redox Biology 12:246-263. 10.1016/j.redox.2017.02.011

Lehmann S, Loh SH, and Martins LM. 2017. Enhancing $\mathrm{NAD}^{+}$salvage metabolism is neuroprotective in a PINK1 model of Parkinson's disease. Biology Open 6:141-147. 10.1242/bio.022186

Liu J, Yang B, Zhou P, Kong Y, Hu W, Zhu G, Ying W, Li W, Wang Y, and Li S. 2017. Nicotinamide adenine dinucleotide suppresses epileptogenesis at an early stage. Scientific Reports 7:7321. 10.1038/s41598-017-07343-0

Magni G, Amici A, Emanuelli M, Orsomando G, Raffaelli N, and Ruggieri S. 2004. Enzymology of $\mathrm{NAD}^{+}$homeostasis in man. Cellular and Molecular Life Sciences 61:19-34. 
324

325

326

327

328

329

330

331

332

333

334

335

336

337

338

339

340

341

342

343

Mills KF, Yoshida S, Stein LR, Grozio A, Kubota S, Sasaki Y, Redpath P, Migaud ME, Apte RS, Uchida K, Yoshino J, and Imai SI. 2016. Long-Term Administration of Nicotinamide Mononucleotide Mitigates Age-Associated Physiological Decline in Mice. Cell Metabolism 24:795-806. 10.1016/j.cmet.2016.09.013

Rani V, Deep G, Singh RK, Palle K, and Yadav UC. 2016. Oxidative stress and metabolic disorders: Pathogenesis and therapeutic strategies. life sciences 148:183-193. 10.1016/j.lfs.2016.02.002

Revollo JR, Grimm AA, and Imai S. 2007. The regulation of nicotinamide adenine dinucleotide biosynthesis by Nampt/PBEF/visfatin in mammals. Current Opinion in Gastroenterology 23:164-170. 10.1097/MOG.0b013e32801b3c8f

Sheng C, Chen H, Wang B, Liu T, Hong Y, Shao J, He X, Ma Y, Nie H, Liu N, Xia W, and Ying W. 2012. $\mathrm{NAD}^{+}$administration significantly attenuates synchrotron radiation X-rayinduced DNA damage and structural alterations of rodent testes. International journal of physiology, pathophysiology and pharmacology 4:1-9.

Son MJ, Kwon Y, Son T, and Cho YS. 2016. Restoration of Mitochondrial NAD ${ }^{+}$Levels Delays Stem Cell Senescence and Facilitates Reprogramming of Aged Somatic Cells. Stem Cells 34:2840-2851. 10.1002/stem.2460

Ureshino RP, Rocha KK, Lopes GS, Bincoletto C, and Smaili SS. 2014. Calcium signaling alterations, oxidative stress, and autophagy in aging. Antioxidants \& Redox Signaling 21:123-137. 10.1089/ars.2013.5777 
344 Wang B, Ma Y, Kong X, Ding X, Gu H, Chu T, and Ying W. 2014. NAD ${ }^{+}$administration decreases

345 doxorubicin-induced liver damage of mice by enhancing antioxidation capacity and 346 decreasing DNA damage. Chemico-Biological Interactions 212:65-71. 10.1016/j.cbi.2014.01.013

348 Won SJ, Choi BY, Yoo BH, Sohn M, Ying W, Swanson RA, and Suh SW. 2012. Prevention of traumatic brain injury-induced neuron death by intranasal delivery of nicotinamide adenine dinucleotide. Journal of Neurotrauma 29:1401-1409. 10.1089/neu.2011.2228

Ying W. 2008. NAD + NADH and NADP ${ }^{+} / \mathrm{NADPH}$ in cellular functions and cell death: regulation and biological consequences. Antioxidants \& Redox Signaling 10:179-206. 10.1089/ars.2007.1672

Ying W, Wei G, Wang D, Wang Q, Tang X, Shi J, Zhang P, and Lu H. 2007. Intranasal administration with $\mathrm{NAD}^{+}$profoundly decreases brain injury in a rat model of transient focal ischemia. Frontiers in Bioscience 12:2728-2734. 10.2741/2267

Ying WH. 2006. NAD ${ }^{+}$and NADH in cellular functions and cell death. Frontiers in Bioscience 11:3129-3148. Doi 10.2741/2038

Yoshino J, Mills KF, Yoon MJ, and Imai S. 2011. Nicotinamide mononucleotide, a key NAD ${ }^{+}$ intermediate, treats the pathophysiology of diet- and age-induced diabetes in mice. Cell Metabolism 14:528-536. 10.1016/j.cmet.2011.08.014

Zhang MC, and Ying WH. 2019. NAD ${ }^{+}$Deficiency Is a Common Central Pathological Factor of a Number of Diseases and Aging: Mechanisms and Therapeutic Implications. Antioxidants \& Redox Signaling 30:890-905. 10.1089/ars.2017.7445 
365 Zhang Y, Wang B, Fu X, Guan S, Han W, Zhang J, Gan Q, Fang W, Ying W, and Qu X. 2016. 366 Exogenous $\mathrm{NAD}^{+}$administration significantly protects against myocardial 367 ischemia/reperfusion injury in rat model. American Journal of Translational Research 368 8:3342-3350.

Zheng C, Han J, Xia W, Shi S, Liu J, and Ying W. 2012. NAD ${ }^{+}$administration decreases ischemic brain damage partially by blocking autophagy in a mouse model of brain ischemia. Neuroscience Letters 512:67-71. 10.1016/j.neulet.2012.01.007

Zuo L, Prather ER, Stetskiv M, Garrison DE, Meade JR, Peace TI, and Zhou T. 2019. Inflammaging and Oxidative Stress in Human Diseases: From Molecular Mechanisms to 


\section{Figure 1}

Fig. 1. Nampt plays a siginificant role in the $N A D^{+}$synthesis and basal survival of differentiated PC12 cells.

(A) FK866 at the concentrations of 2, 5, 10, 20, and $50 \mathrm{nM}$ produced dose-dependent decreases in the intracellular $\mathrm{NAD}^{+}$levels. $\mathrm{NAD}^{+}$assays were conducted $6 \mathrm{hrs}$ after the cells were treated with $2,5,10,20$, or $50 \mathrm{nM}$ FK866. The data were pooled from three independent experiments. $N=3$. There were 3,3 and 3 samples in these three independent experiments. $* * *, P<0.001$. (B,C) Ten nM FK866 led to a significant increase in early-stage apoptosis, while both $20 \mathrm{nM}$ and $50 \mathrm{nM}$ FK866 led to significant increases in early-stage apoptosis (Annexin $\mathrm{V}^{+} / 7-\mathrm{ADD}^{-}$), late-stage apoptosis (Annexin $\mathrm{V}^{+} / 7-\mathrm{ADD}^{+}$) and necrosis (Annexin V/7-ADD ). PC12 cells were treated with 10, 20, or 50 nM FK866 for 24 hrs. The data were pooled from three independent experiments. $N=3$. There were 3,3 and 3 samples in these three independent experiments. $* *, P<0.01 ; * * *, P<0.001$. 


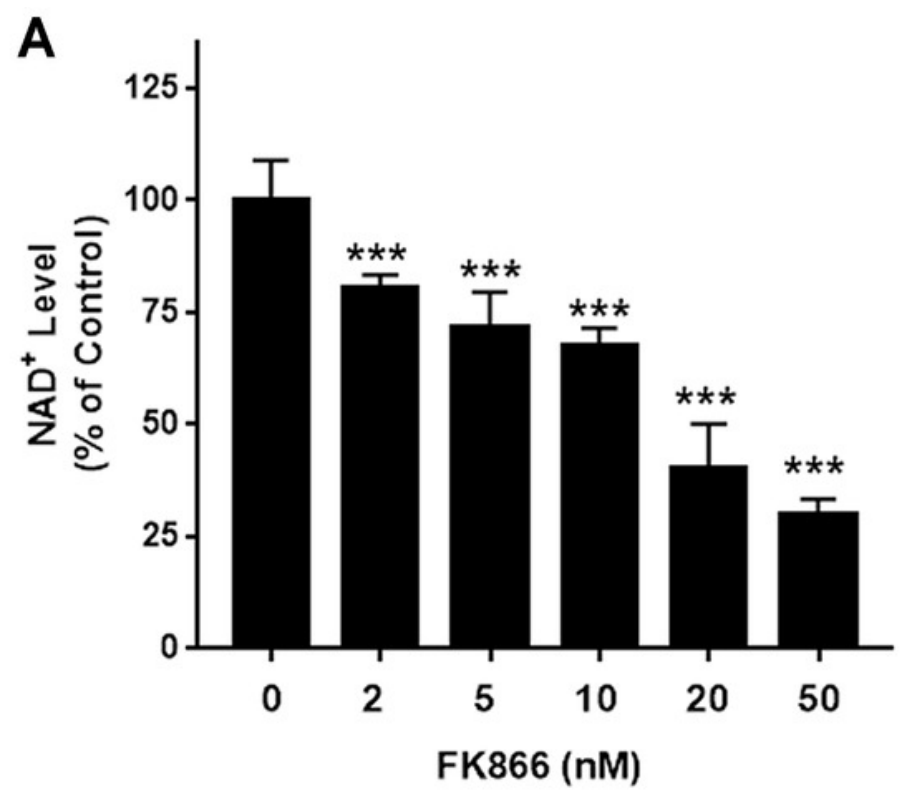

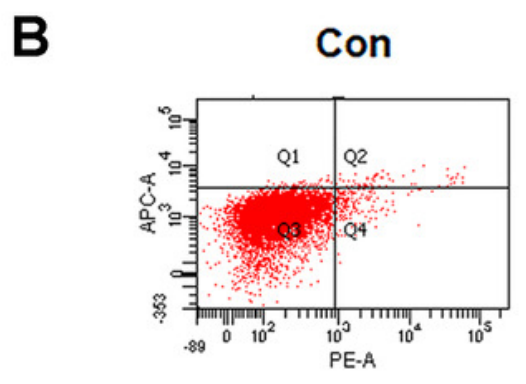

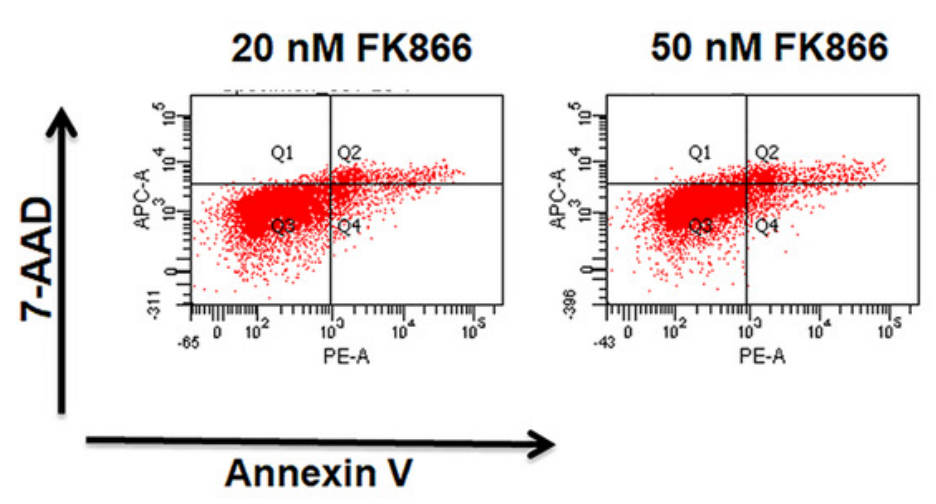

C

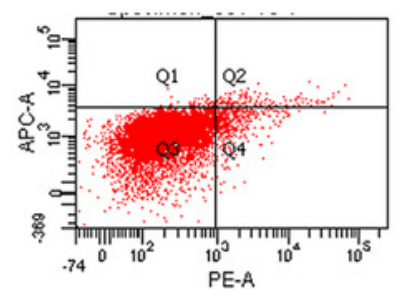

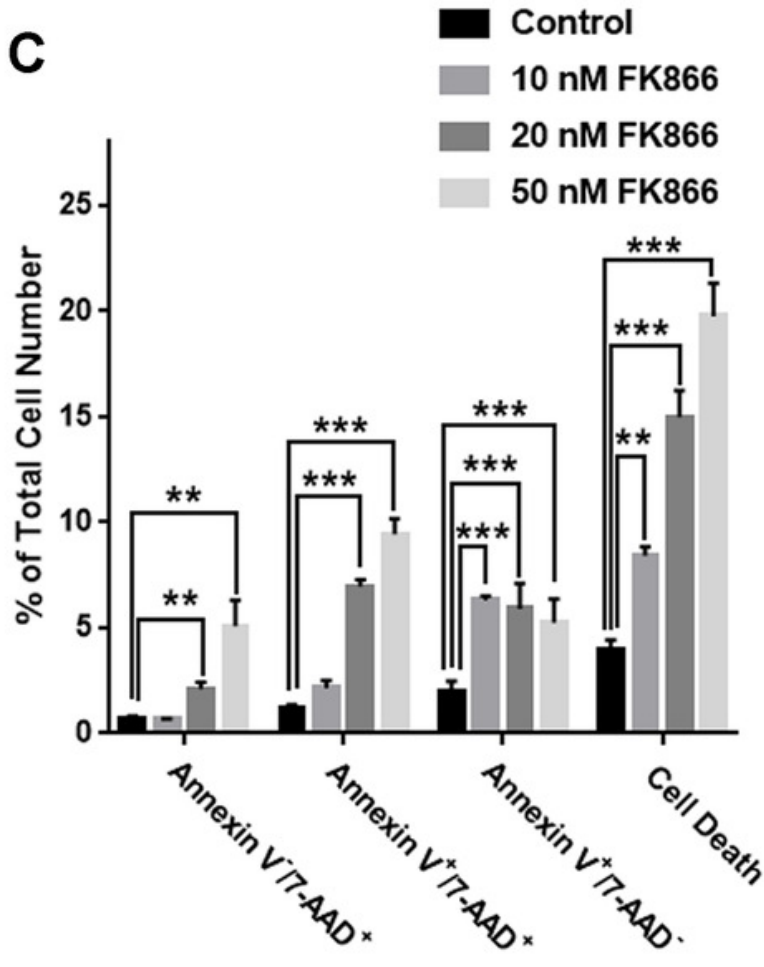




\section{Figure 2}

Fig. 2. $\mathrm{H}_{2} \mathrm{O}_{2}$ produced significant decreases in both Nampt mRNA levels and Nampt protein levels of PC12 cells.

(A) Treatment of PC12 cells with $0.1 \mathrm{mM}$ or $0.3 \mathrm{mM} \mathrm{H}_{2} \mathrm{O}_{2}$ led to significant increases in the Nampt mRNA level of the cells 12 hrs after the $\mathrm{H}_{2} \mathrm{O}_{2}$ treatment. In contrast, $\mathrm{H}_{2} \mathrm{O}_{2}$ led to significant decreases in the Nampt mRNA level of the cells $20 \mathrm{hrs}$ after the $\mathrm{H}_{2} \mathrm{O}_{2}$ treatment. PC12 cells were treated with 0.1 or $0.3 \mathrm{mM} \mathrm{H}_{2} \mathrm{O}_{2}$ for 12 or $20 \mathrm{hrs}$. The data were pooled from three independent experiments. $N=3$. There were 3,3 and 3 samples in these three independent experiments. $*, P<0.05 ; * *, P<0.01$. (B) Western blot assays showed the $\mathrm{e}$ ffects of $\mathrm{H}_{2} \mathrm{O}_{2}$ on the protein level of Nampt $12 \mathrm{hrs}$ after the $\mathrm{H}_{2} \mathrm{O}_{2}$ treatment. (C) Western blot assays showed the effects of $\mathrm{H}_{2} \mathrm{O}_{2}$ on the protein level of Nampt 24 hrs after the $\mathrm{H}_{2} \mathrm{O}_{2}$ treatment. (D) Western blot assays showed the effects of $\mathrm{H}_{2} \mathrm{O}_{2}$ on the protein level of Nampt $48 \mathrm{hrs}$ after the $\mathrm{H}_{2} \mathrm{O}_{2}$ treatment. (E) Quantifications of the Western blot assays showed that $\mathrm{H}_{2} \mathrm{O}_{2}$ led to significant decreases in the Nampt protein level of the cells 24 and $48 \mathrm{hrs}$, but not $12 \mathrm{hrs}$, after the $\mathrm{H}_{2} \mathrm{O}_{2}$ treatment. $\mathrm{PC} 12$ cells were treated with 0.1 or $0.3 \mathrm{mM} \mathrm{H}_{2} \mathrm{O}_{2}$ for 12,24 , or $48 \mathrm{hrs}$. The data were pooled from three independent experiments. $N=3$. There were 3,3 and 6 samples in these three independent experiments. $*, P<0.05 ; * *, P<0.01$. 


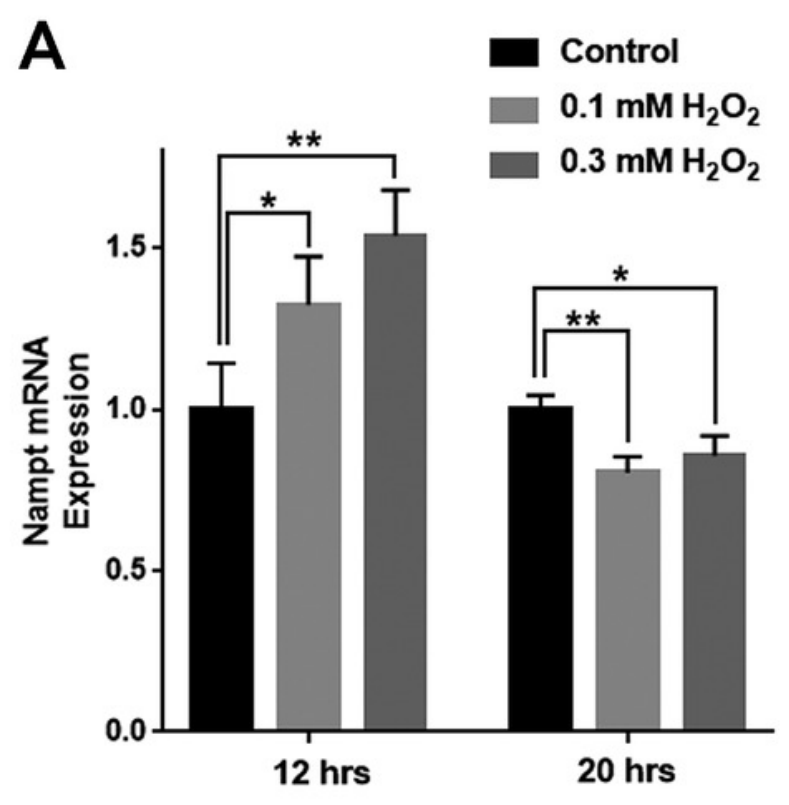

B

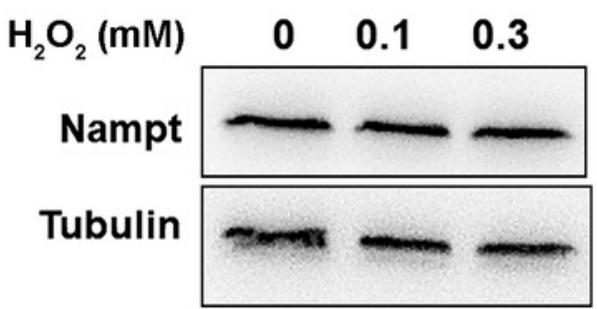

E

Control

$0.1 \mathrm{mM} \mathrm{H}_{2} \mathrm{O}_{2}$

$12 \mathrm{hrs}$

C

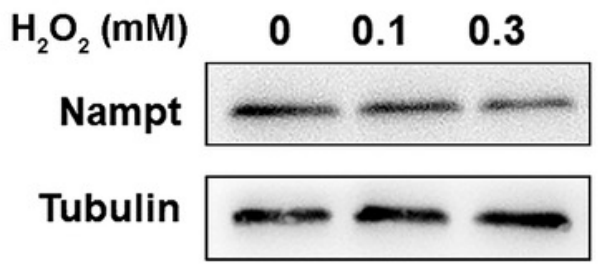

D
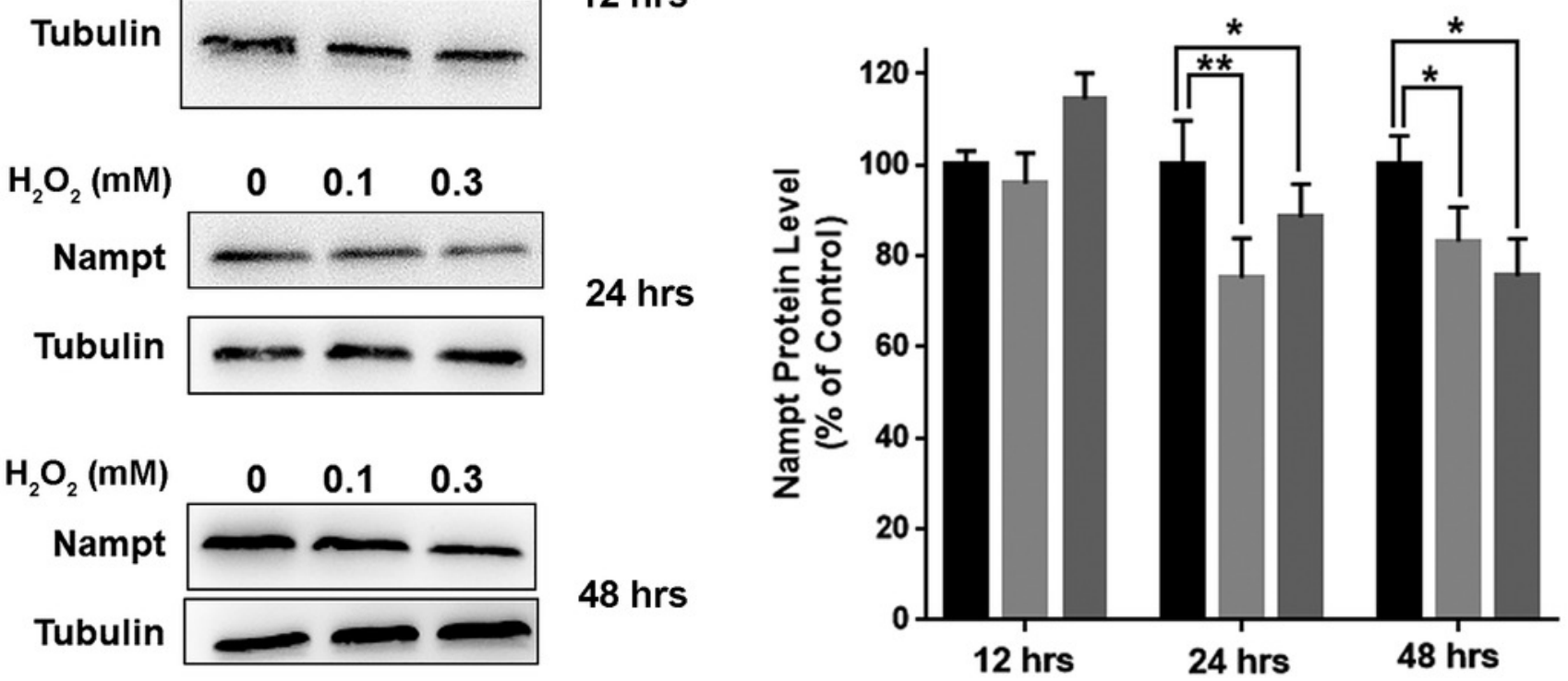


\section{Figure 3}

Fig. 3. Nampt siRNA and FK866 significantly exacerbated $\mathrm{H}_{2} \mathrm{O}_{2}$-produced decreases in the intracellular $\mathrm{NAD}^{+}$levels and cell survival of differentiated PC12 cells.

(A, B) Treatment of the cells with Nampt siRNA led to a significant decrease in the Nampt protein level. PC12 cells were transfected with control or Nampt siRNA sequences for $48 \mathrm{hrs}$. There were 12 samples in the experiments. (C) Nampt siRNA treatment significantly exacerbated $\mathrm{H}_{2} \mathrm{O}_{2}$-produced decrease in the NAD levels of PC12 cells. (D, E) Nampt siRNA significantly exacerbated $\mathrm{H}_{2} \mathrm{O}_{2}$-produced late-stage apoptosis (Annexin $\mathrm{V}^{+} / 7-\mathrm{ADD}^{+}$) and necrosis (Annexin V/7-ADD ${ }^{+}$) of the cells. PC12 cells were transfected with control or Nampt siRNA sequences for $24 \mathrm{hrs}$, and then co-treated with $0.3 \mathrm{mM} \mathrm{H}_{2} \mathrm{O}_{2}$ for 6 hrs (for $\mathrm{NAD}^{+}$assay) or $24 \mathrm{hrs}$ (for flow cytometry assay). The data were pooled from three independent experiments. $N=3$. There were 3, 3 and 3 samples in these three independent experiments. $*, P<0.05 ; * *, P<0.01 ; * * *, P<0.001$. (F) FK866 significantly exacerbated $\mathrm{H}_{2} \mathrm{O}_{2}-$ produced decrease in the NAD levels of PC12 cells. (G, H) FK866 significantly exacerbated $\mathrm{H}_{2} \mathrm{O}_{2}$-produced early-stage apoptosis (Annexin $\mathrm{V}^{+} / 7-\mathrm{ADD}^{-}$), late-stage apoptosis (Annexin $\mathrm{V}^{+} / 7-\mathrm{ADD}^{+}$) and necrosis (Annexin $\mathrm{V}^{-} / 7-\mathrm{ADD}^{+}$) of the cells. PC12 cells were pre-treated with 10 nM FK866 for 30 min, and then co-treated with $0.3 \mathrm{mM} \mathrm{H}_{2} \mathrm{O}_{2}$ for 6 hrs (for NAD ${ }^{+}$assay) or 23.5 hrs (for flow cytometry assay). The data were pooled from three independent experiments. $N$ $=3$. There were 3,3 and 3 samples in these three independent experiments. $*, P<0.05$; $* *, P<0.01 ; * * *, P<0.001$. 


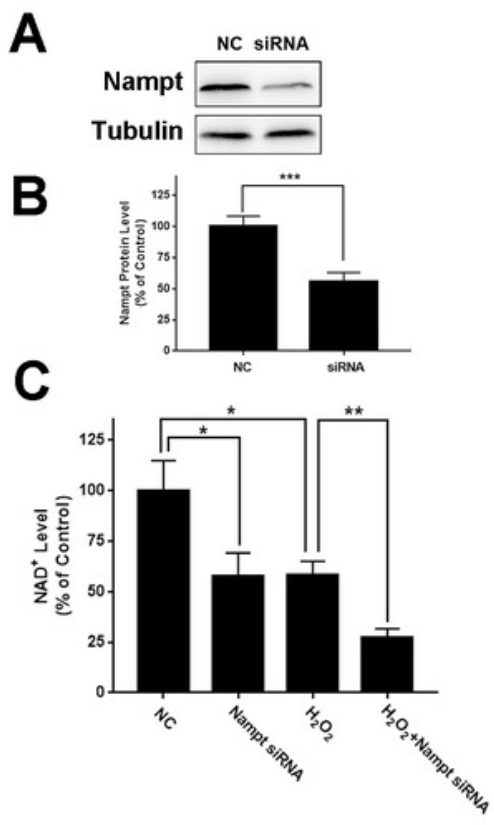

$\mathbf{F}$

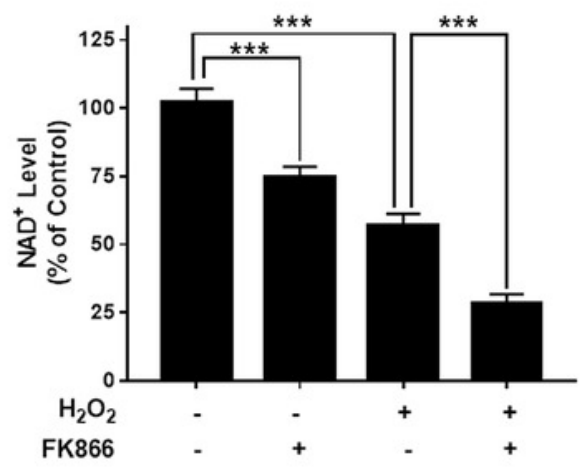

D
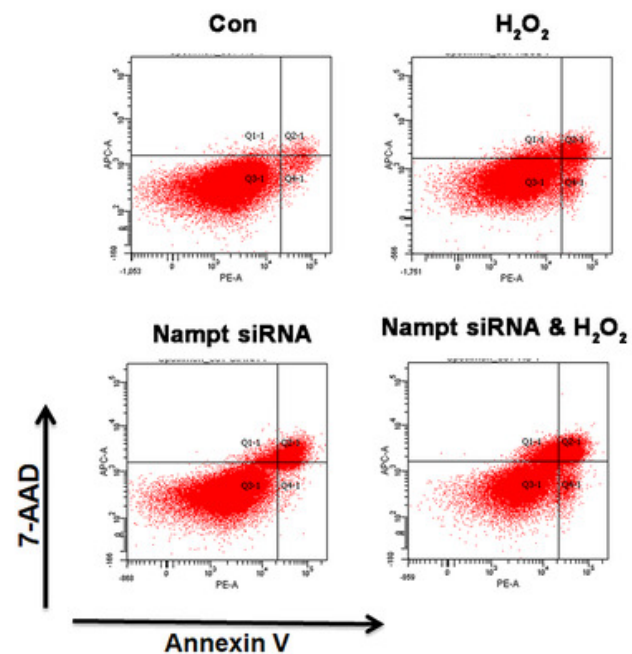

G
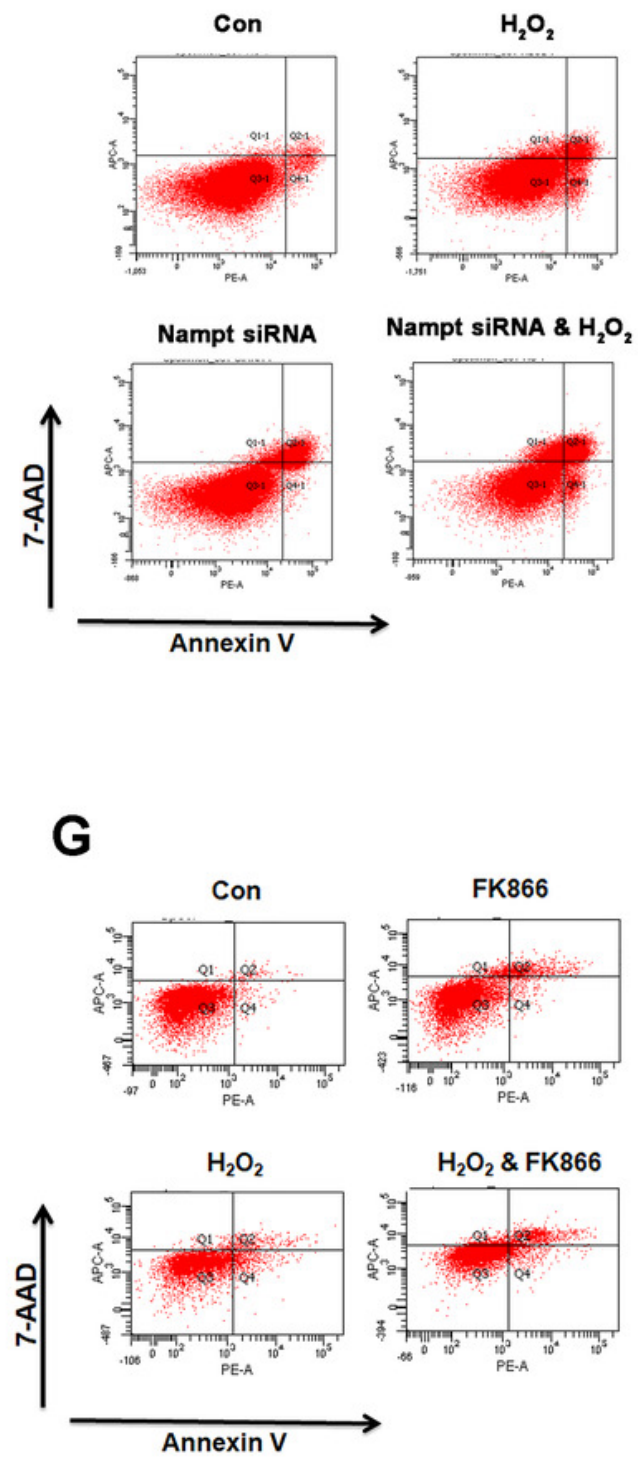

E

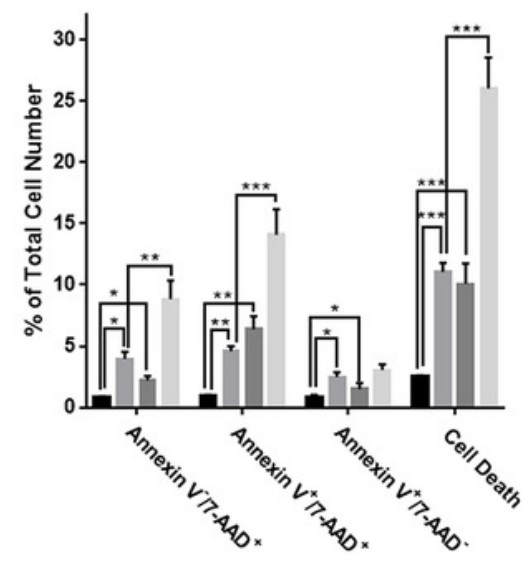

H
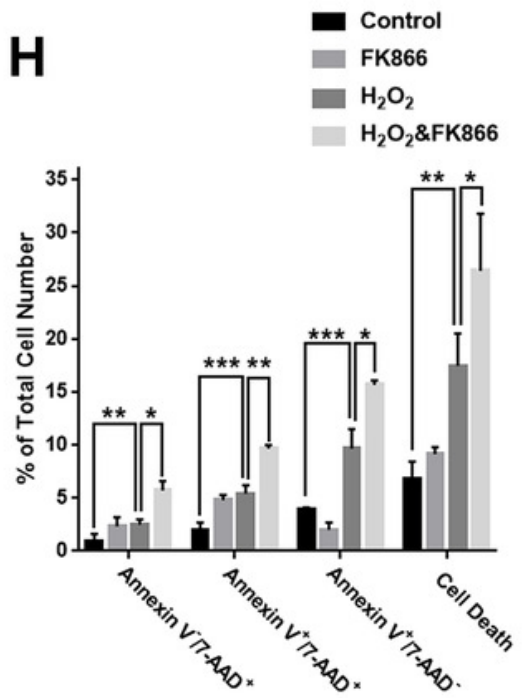


\section{Figure 4}

Fig. 4. Nampt activator P7C3 significantly attenuated $\mathrm{H}_{2} \mathrm{O}_{2}$-produced decreases in the intracellular $\mathrm{NAD}^{+}$levels and cell survival of differentiated PC12 cells.

(A) P7C3 significantly attenuated the $\mathrm{H}_{2} \mathrm{O}_{2}$-produced decrease in the intracellular $\mathrm{NAD}^{+}$levels of PC12 cells. (B, C) Nampt activator P7C3 significantly attenuated the $\mathrm{H}_{2} \mathrm{O}_{2}$-produced earlystage apoptosis (Annexin $\mathrm{V}^{+} / 7-\mathrm{ADD}^{-}$) of the cells. PC12 cells were pre-treated with $10 \mathrm{mM}$ P7C3 for 4 hrs, and then co-treated with $0.3 \mathrm{mM} \mathrm{H}_{2} \mathrm{O}_{2}$ for 6 hrs (for NAD assay) or 20 hrs (for flow cytometry assay). The data were pooled from three independent experiments. $N=3$. There were 3, 3 and 3 samples in these three independent experiments. $*, P<0.05 ; * *, P$ $<0.01 ; * * *, P<0.001$. 


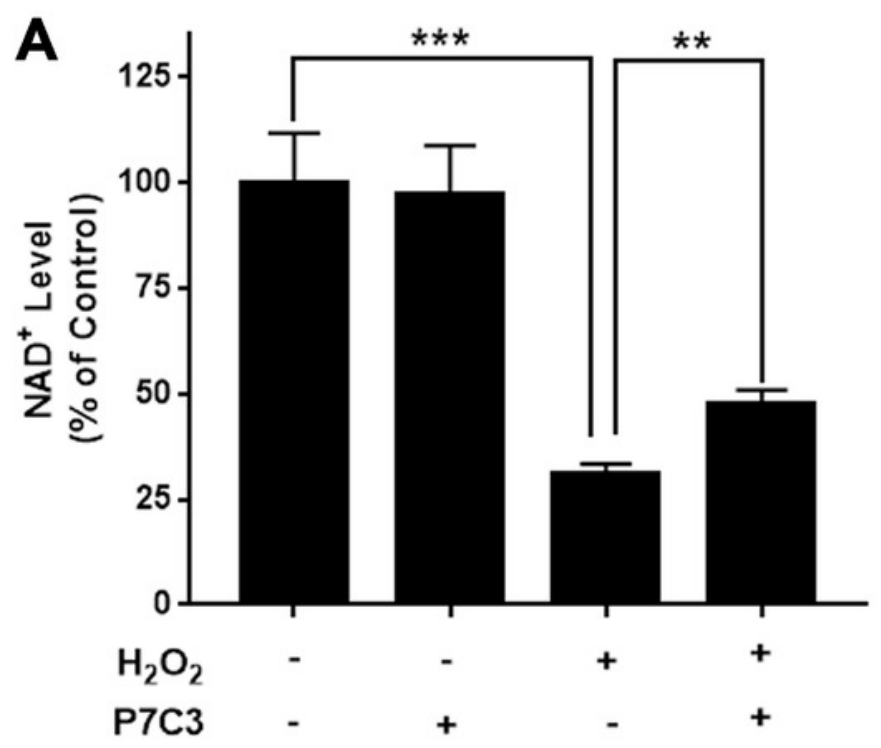

B
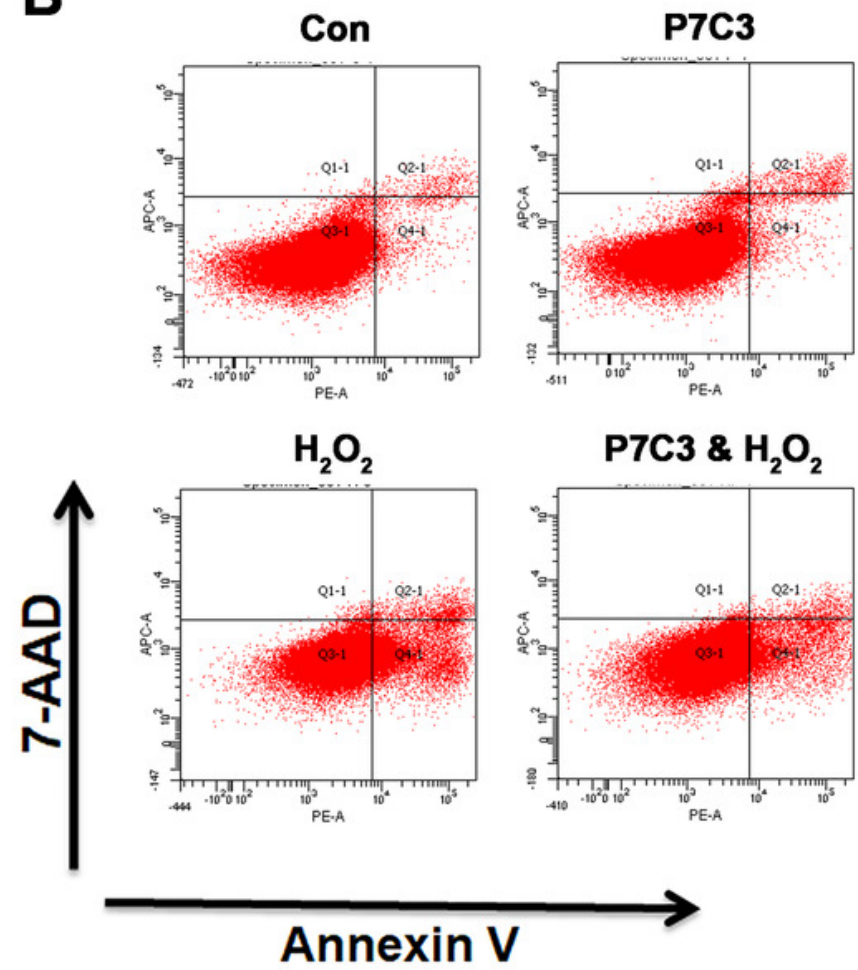

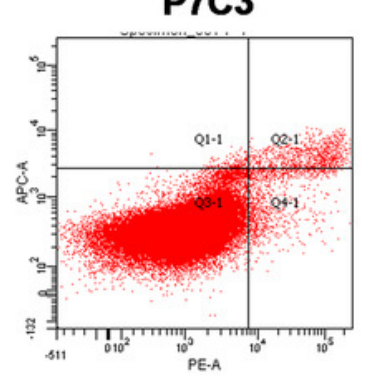

Annexin V
C

Control

P7C3

$\mathrm{H}_{2} \mathrm{O}_{2}$

P7C3 \& $\mathrm{H}_{2} \mathrm{O}_{2}$

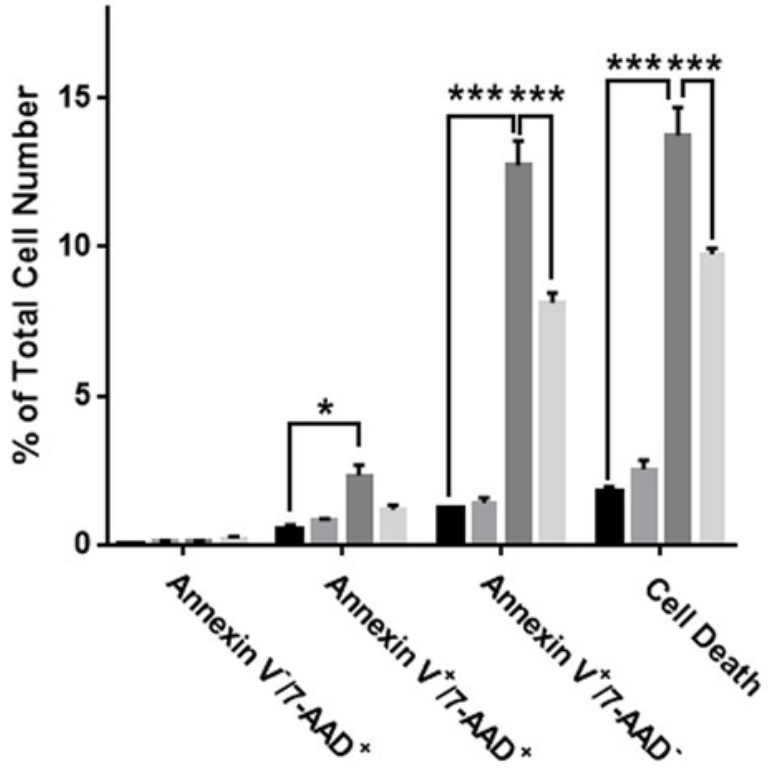

